\title{
IDEAS SOBRE LA PERMEABILIDAD EN ESTUDIANTES DE MAGISTERIO
}

\author{
Cort és Gracia, Ángel Luis \\ Departamento de Didáctica de las Ciencias Experimentales. Facultad de Educación. Universidad de Zaragoza \\ San Juan Bosco, 7.50009 Zaragoza \\ acortes@posta.unizar.es
}

\begin{abstract}
Resumen. El análisis de las ideas sobre permeabilidad que presentan los estudiantes de magisterio muestra la existencia de diversas concepciones alternativas y esquemas conceptuales. Estas ideas alternativas no son consistentes con el significado semántico y científico del término. Las causas deben buscarse, entre otras, en la formación previa de los estudiantes, con un uso del término en la vida cotidiana diferente del científico y a la existencia de libros de texto con definiciones y modelos erróneos.
\end{abstract}

Palabras clave. Permeabilidad, ciencia escolar, formación de profesores, concepciones alternativas, libros de texto.

Summary. The analysis of students' ideas about permeability was carried out among students from a university teacher training course. It shows several alternative conceptions and conceptual frameworks. Obstacles can be due, among others, to a different daily use of this concept during previous to a learning with respect to the scientific use and to incorrect definitions and models included in some textbooks.

K eywords. Permeability, school science, teacher training, alternative conceptions, textbooks.

\section{INTRODUCCIÓN}

La investigación sobre conocimientos e ideas previas, representaciones mentales, concepciones alternativas, etc. ha alcanzado un gran desarrollo dentro del modelo constructivista de enseñanza-aprendizaje de las ciencias (Driver 1988; Driver et al., 1989; Furió Más, 1996; Pozo, 1996; Prieto y Blanco, 1997). En muchas ocasiones, los errores se han recogido como ejemplos divertidos o chocantes, si bien es necesario analizar estos problemas en profundidad, ya que en algunos casos representan un alto porcentaje de las concepciones de los estudiantes (Pozo y Gómez Crespo, 1998). Cuando los conceptos o los modelos científicos se mezclan con aspectos de la vida cotidiana, aparecen concepciones alternativas que, frecuentemente, constituyen verdaderas «teorías implícitas» (Pozo y Gómez Crespo, 1998), bastante diferentes e incluso enfrentadas a las «teorías científicas» comúnmente aceptadas.

El tema «Los materiales y sus propiedades» constituye uno de los ejes en torno a los que se articula la enseñanza-aprendizaje de las ciencias desde los primeros niveles educativos y es la base para comprender muchos otros contenidos del currículo oficial, como los relacionados con el paisaje, el medio físico, los seres vivos, etc. Las concepciones de los estudiantes sobre la naturaleza de la materia y las diferentes propiedades de los materiales han dado lugar a numerosos trabajos en los últimos años (Krnel et al., 1998; Prieto et al., 2000; entre otros).

En el marco de la enseñanza-aprendizaje de las ciencias naturales (y específicamente en geología) es necesario un correcto conocimiento de las propiedades de los materiales (sedimentos, rocas, suelos) para la comprensión de muchos fenómenos geológicos en los que se encuentran implícitas. Podemos tomar como ejemplo las referencias de Yus Ramos y Rebollo (1993), sobre la formación de suelos, o la de Yus Ramos (1994), sobre la circulación freática.

Yus Ramos (1994) aprecia un «importante bloqueo de tipo físico» en el modelo que los estudiantes de $1^{\circ}$ de BUP poseían sobre los mecanismos de circulación 
freática en medios porosos. La investigación sobre las representaciones de los estudiantes es clara y las estrategias didácticas están directamente encaminadas para propiciar un cambio conceptual mediante diálogos socráticos, formulación de hipótesis y modelos analógicos. En el tratamiento didáctico del problema aparece continuamente de forma implícita o explícita el concepto de permeabilidad. El autor mencionado utiliza modelos con medios permeables e impermeables que podría ayudar a un aprendizaje significativo de los procesos de circulación freática.

Sin embargo, en nuestro trabajo nos planteamos varias cuestiones: ¿Conocen realmente los estudiantes el significado del concepto permeabilidad? ¿Utilizan correctamente, desde el punto de vista científico, los términos permeable e impermeable al hablar de los elementos del medio natural? ¿Saben por qué los distintos materiales son más o menos permeables?

En este trabajo mostramos un estudio llevado a cabo con estudiantes de magisterio, sometiendo a su consideración un problema visual sobre tres situaciones distintas y planteando una serie de cuestiones sobre el mismo. Los resultados recogidos en tablas y debidamente comentados, ponen en evidencia la dificultad del concepto permeabilidad. Veamos a continuación el esquema metodológico empleado, los resultados obtenidos y su interpretación.

\section{PROCESO METODOLÓGICO}

\section{Algunas de las dificultades del concepto permeabi- lidad}

El concepto de permeabilidad aparece íntimamente ligado a la enseñanza de las ciencias en los distintos niveles educativos, desde la enseñanza primaria hasta la universitaria. Se encuentra implícito en muchas actividades de física y química. Es utilizado explícitamente en biología para explicar los procesos de ósmosis (paso del disolvente a través de membranas semipermeables...), de germinación (la cubierta de las semillas es permeable al agua...), etc. En geología, es un concepto imprescindible para poder comprender una gran cantidad de hechos y procesos superficiales y profundos, desde la formación de un charco en la calle hasta la existencia de yacimientos de hidrocarburos, como el petróleo, o la comprensión de los sistemas hidrogeológicos subterráneos, así como la manera en que éstos se recargan de agua y descargan la misma en superficie.

En la vida cotidiana el concepto de permeabilidad surge constantemente. De hecho, buena parte de las personas se viste con un impermeable cuando llueve, otros impermeabilizan el tejado de sus casas para evitar goteras, o el fondo de su balsa o piscina para evitar pérdidas de agua.

Según el Diccionario de la Real Academia Española de la Lengua (RAE, 2001), permeable procede del latín permeabilis, penetrable, y hace referencia a aquello «que pue- de ser penetrado o traspasado por el agua u otro fluido». Permeabilidad, por tanto, es cualidad de permeable.

De este modo, que un material sea permeable implica que puede ser traspasado por un fluido, y esto, a su vez, implica que el fluido entra en el cuerpo permeable y, posteriormente, sale de éste. En relación con este significado, y como aproximación, se asume que un cuerpo es tanto más permeable cuanta más cantidad de fluido deje pasar. Así, podríamos diferenciarlo del concepto de absorción, en el que el fluido entra debido a la atracción que ejerce el cuerpo absorbente, pero no necesariamente tiene que salir del mismo.

Desde el punto de vista científico, el concepto es relativamente más complejo. Por ejemplo en hidráulica (Custodio y Llamas, 1976; Price, 1996, etc.), la ley de Darcy define la permeabilidad o conductividad hidráulica $(K)$ como la «capacidad de una roca para que un fluido fluya a través de ella» y se mide en darcys (que equivalen a la permeabilidad de un fluido de 1 centipoise de viscosidad que fluye a una velocidad de $1 \mathrm{~cm} / \mathrm{s}$ a una presión de 1 $\mathrm{atm} / \mathrm{cm})$. Es decir, es la expresión de la facilidad con que un fluido atraviesa un material poroso y se puede calcular a partir del volumen de fluido que atraviesa una longitud de roca o suelo de sección constante en un tiempo dado. Como citan Prieto y Blanco (1997), algunos conceptos científicos «precisan de aparato matemático para ser correctamente expresados». De esta forma, la expresión matemática de la permeabilidad sería:

siendo:

$$
q=-K(d h / d l)
$$

$q=\mathrm{Q} /$ sección (caudal que circula por metro cuadrado de sección) $K=$ Conductividad hidráulica (mejor que permeabilidad, según numerosas referencias)

$d h / d l=$ gradiente hidráulico (diferencia de potencial entre dos puntos en relación con la distancia entre los mismos)

Al margen de la formulación matemática del concepto, lo que podemos percibir directamente es el paso del fluido (agua, por ejemplo) a través del material. También podemos medir el tiempo que tarda en pasar una determinada cantidad de agua o la cantidad de agua que pasa en un tiempo dado a través del material permeable. Difícilmente podemos observar (al menos en la observación cotidiana) el paso del agua a través de los «espacios vacíos» que contiene el material (huecos, poros, conductos, etc.).

Se pueden distinguir, al menos, dos categorías ontológicas alrededor del concepto citado (en el sentido de Chi et al., 1994; Prieto y Blanco, 1997):

1) La primera correspondería a la «materia», es decir, la permeabilidad es una propiedad de la materia, aunque no sea tan evidente como la forma o el color.

2) La segunda hace referencia a un «proceso», como es el paso del fluido a través del material, y que depende del resto de las propiedades del material y de las del fluido. 
Si compartimos unos criterios convencionales sobre colores y formas, diremos que un objeto es blanco y tiene una forma determinada simplemente viendo una imagen del mismo. Para saber, además, si es o no permeable deberemos probar a «echarle agua» y esperar a que pase.

Aunque existen algunas propuestas didácticas para trabajar el concepto de permeabilidad en las aulas como la de Lillo (1983), que trata el tema de forma explícita, y la de Yus Ramos (1994), que lo hace de forma implícita, en general se trata de un término que, asumimos, no debería presentar demasiadas dificultades en el proceso de enseñanzaaprendizaje. Quizá por ello, ya en los textos de educación primaria es frecuente ver actividades relacionadas implícita o explícitamente con el término, pero no siempre aparece una explicación acertada sobre el mismo. O dicho de otra manera, el modelo presentado dista mucho del científico y, en muchas ocasiones, ni siquiera se acerca a la definición del diccionario. De este modo, en la mayoría de los casos queda en manos del profesor que los estudiantes consigan un aprendizaje significativo de este concepto.

\section{Elección de la muestra sometida a experiencia y grupos de control}

La experiencia se ha realizado con un total de 147 estudiantes de la diplomatura de maestro. La primera muestra consta de 90 estudiantes que corresponden a dos grupos de 45 estudiantes de tercer curso de la especialidad de educación primaria (MEP). Ambos grupos realizaron la misma prueba en los cursos 1999-2000 (grupo M1) y 2001-2002 (grupo M2). Los dos presentaban unas características formativas previas muy similares. Se planteaba un problema dentro de un contexto general de indagación en el aula y a partir de esa prueba (que aquí aparece como «cuestionario») se detectaron esas ideas. El profesor fue el mismo en ambos casos y la prueba se realizó sin una preparación previa específica y explícita sobre el concepto de permeabilidad. Éste había surgido, sin embargo, en numerosas ocasiones de forma implícita en el aula, ya que los estudiantes habían trabajado con filtros de arena y grava, habían cultivado plantas en distintos sustratos, habían utilizado plásticos para impermeabilizar sus preparaciones, etc.

Tras un primer análisis de los resultados se procedió al contraste de los mismos con tres grupos de control durante el curso 2002-2003. El primer grupo (C1) corresponde a 20 estudiantes del mismo curso y especialidad $\left(3^{\circ} \mathrm{MEP}\right)$ en las mismas condiciones de preparación. El segundo grupo (C2) corresponde a 21 estudiantes del mismo curso y especialidad ( $\left.3^{\circ} \mathrm{MEP}\right)$ que habían trabajado, en otra asignatura, una unidad didáctica sobre los materiales y sus propiedades (aunque no específica sobre permeabilidad). El tercer grupo (C3) corresponde a 16 estudiantes de tercer curso de la especialidad de audición y lenguaje ( $\left.3^{\circ} \mathrm{MAL}\right)$, sin preparación previa específica. Estos tres grupos de control sólo debían responder a las respuestas $\mathrm{F}$ y $\mathrm{G}$ del cuestionario que veremos más adelante. A continuación mostraremos las ideas que presentan estos estudiantes en relación con el concepto analizado.

\section{Formulación del problema}

- Imagina que tienes tres recipientes exactamente iguales (perforados por el fondo) en los que introduces respectivamente: a) grava gruesa, b) grava fina y c) arena de playa (volúmenes iguales). El montaje se muestra en la figura adjunta.

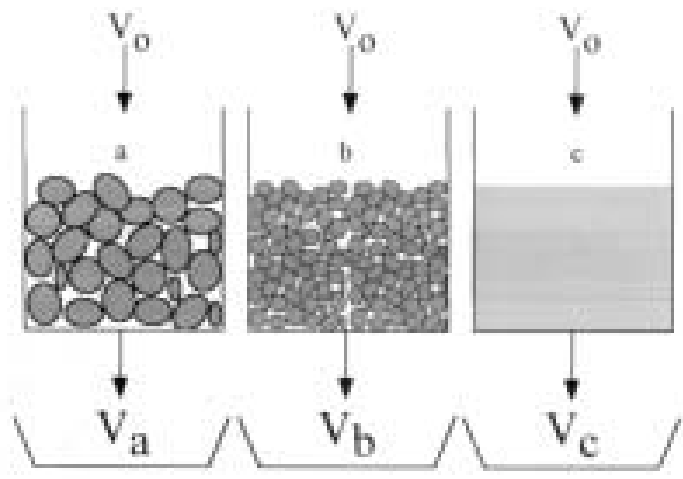

- Si echas la misma cantidad de agua (Vo) en cada recipiente, al cabo de un tiempo habrán pasado las siguientes cantidades de agua: $V a>V b>V c$.

Ante el problema planteado, los estudiantes debían responder a las siguientes cuestiones abiertas relacionadas con la permeabilidad. Entre las preguntas que hacían referencia directa a la misma se intercalaban otras generales relacionadas con la propia actividad experimental, de manera que no era un cuestionario exclusivamente dirigido hacia el concepto citado.

\section{Cuestiones}

A. ¿Cuál es el fenómeno(s) observado(s)?

B. ¿Qué otros conceptos (además de permeabilidad) están relacionados?

C. ¿Qué tipos de registros o medidas se pueden realizar?

D. ¿Qué variables aparecen? (diferencia entre dependientes e independientes).

E. ¿Qué conclusiones se pueden obtener a partir de los resultados experimentales?

F. ¿Cuál de los tres materiales es más permeable? ¿Cómo podrías definir la permeabilidad?

G. Busca alguna analogía de la vida cotidiana para ejemplificar el fenómeno o el concepto.

Las preguntas señaladas en cursiva podían ser contestadas o justificadas teóricamente sin la ayuda del dispositivo experimental y sus resultados, que han actuado para algunos estudiantes como ayudas y en otros, como distractores. Nos hemos centrado en el análisis de las respuestas a estas tres cuestiones, ya que nos permitían detectar las ideas alternativas en torno al concepto analizado. 


\section{Análisis de los resultados obtenidos sobre las cues- tiones B, F y G}

Pregunta B: ¿Qué otros conceptos (además de permeabilidad) están relacionados?

El objetivo de esta pregunta era aproximarnos al contexto teórico (o conceptual) en el que los estudiantes utilizan el término permeabilidad, invitándoles a citar otros conceptos relacionados. Se han obtenido 241 respuestas que hacían referencia a 43 conceptos distintos (Tabla I). Se ha eliminado de entrada el término permeabilidad, ya que éste aparece en el propio cuestionario. En algunos casos, los estudiantes aportaban una breve explicación sobre los conceptos y su relación con el fenómeno observado, aunque la mayoría se limitaba a dar una lista de términos. El gran número y variedad de las respuestas dificulta una valoración sobre la representatividad de las mismas. Como se ha comentado anteriormente, la mayoría de los estudiantes no justificaba su respuesta y, en general, estos términos no eran empleados en la ejemplificación posterior del fenómeno (pregunta $G$ ).

Quizá es más fácil destacar algunos términos que han repetido varios estudiantes y que tienen poca o nula relación con el fenómeno observado, al menos en este contexto: humedad, mezclas, evaporación, transpiración, etc. También hay que señalar el significado que atribuyen algunos estudiantes al término densidad: «grado de compactación de los materiales en cada uno de los recipientes» (según aclaraciones en las mismas respuestas y en posteriores entrevistas personales).

Uno de los términos que más se repite paralelamente a un gran porcentaje de errores (o ideas alternativas) es el de $a b$ sorción. Según el Diccionario de la RAE (2001), absorber, dicho de una sustancia, significa «ejercer atracción sobre un fluido con el que está en contacto, de modo que las moléculas de éste penetren en aquélla». Posteriormente veremos que buena parte de los alumnos han relacionado directamente absorción y permeabilidad, construyendo un significado del tipo: «Es más permeable aquella que más absorbe».

Tabla I

R espuestas a la pregunta B: ¿Qué otros conceptos (además de permeabilidad) están relacionados? Nota: los grupos de control C $1, C 2$ y C 3 no han respondido a esta pregunta.

\begin{tabular}{|c|c|c|c|}
\hline Concepto & $\begin{array}{c}\text { Núm. de } \\
\text { repeticiones }\end{array}$ & $\begin{array}{c}\% \text { sobre el total } \\
\text { de alumnos }\end{array}$ & $\begin{array}{c}\% \text { sobre el total } \\
\text { de respuestas }\end{array}$ \\
\hline Volumen & 38 & 42,2 & 15,8 \\
\hline Filtración & 31 & 34,4 & 12,9 \\
\hline Materiales (características de...) & 20 & 22,2 & 8,3 \\
\hline Absorción & 19 & 21,1 & 7,9 \\
\hline Densidad & 16 & 17,8 & 6,6 \\
\hline Tamaño del material (grano) & 15 & 16,7 & 6,2 \\
\hline Porosidad & 8 & 8,9 & 3,3 \\
\hline Tiempo & 8 & 8,9 & 3,3 \\
\hline Composición (sin especificar) & 8 & 8,9 & 3,3 \\
\hline Humedad & 8 & 8,9 & 3,3 \\
\hline Mezclas & 7 & 7,8 & 2,9 \\
\hline Compactación & 6 & 6,7 & 2,5 \\
\hline Impermeabilidad & 5 & 5,5 & 2,1 \\
\hline Agua & 5 & 5,5 & 2,1 \\
\hline Masa & 5 & 5,5 & 2,1 \\
\hline Evaporación & 4 & 4,4 & 1,6 \\
\hline Gravedad & 4 & 4,4 & 1,6 \\
\hline Erosión & 3 & 3,3 & 1,2 \\
\hline Rocas & 3 & 3,3 & 1,2 \\
\hline Viscosidad & 3 & 3,3 & 1,2 \\
\hline Superficie & 2 & 2,2 & 0,8 \\
\hline Transpiración & 2 & 2,2 & 0,8 \\
\hline Otros (1 repetición) & 21 & 23,3 & 8,7 \\
\hline Total respuestas & 241 & & \\
\hline
\end{tabular}


Tabla II

Respuestas a la pregunta $F(1): ~ ¿ C$ uál de los tres materiales es más permeable? (*R espuesta correcta).

\begin{tabular}{|c|c|c|c|c|c|c|c|c|}
\hline Material & $\begin{array}{c}\text { Número } \\
\text { de respuestas } \\
(\text { M1 + M2) }\end{array}$ & $\begin{array}{c}\% \text { total } \\
(\mathrm{M} 1+\mathrm{M} 2)\end{array}$ & $\% \mathrm{M1}$ & $\% \mathrm{M} 2$ & $\begin{array}{c}\text { Número } \\
\text { de respuestas } \\
(\mathbf{C} 1+\mathbf{C} 2+\mathbf{C} 3) \\
\end{array}$ & $\% \mathrm{C} 1$ & $\% \mathrm{C} 2$ & $\% \mathrm{C3}$ \\
\hline$A^{*}$ & 56 & 62,2 & 75,5 & 48,9 & 35 & 55 & 81 & 44 \\
\hline B & 0 & 0 & 0 & 0 & 1 & 5 & 0 & 0 \\
\hline C & 34 & 37,8 & 24,5 & 51,1 & 21 & 40 & 19 & 56 \\
\hline NS/NC & 0 & 0 & 0 & 0 & 0 & 0 & 0 & 0 \\
\hline Total respuestas & 90 & & 45 & 45 & 57 & 20 & 21 & 16 \\
\hline
\end{tabular}

Tabla III

Respuestas a la pregunta F (2): ¿C ómo podrías definir la permeabilidad? (*R espuesta correcta).

\begin{tabular}{|c|c|c|c|c|c|c|c|c|}
\hline Respuesta & $\begin{array}{c}\text { Número } \\
\text { de respuestas } \\
\text { (M1 + M2) }\end{array}$ & $\begin{array}{c}\% \text { total } \\
(\mathrm{M} 1+\mathrm{M} 2)\end{array}$ & $\%$ M1 & $\% \mathrm{M2}$ & $\begin{array}{c}\text { Número } \\
\text { de respuestas } \\
(\mathbf{C} 1+\mathbf{C} 2+\mathbf{C} 3)\end{array}$ & $\% \mathrm{C1}$ & $\% \mathrm{C2}$ & $\% \mathrm{C3}$ \\
\hline $\begin{array}{l}\text { Capacidad para dejar } \\
\text { pasar un líquido...* }\end{array}$ & 53 & 58,9 & 60 & 57,8 & 41 & 70 & 95 & 44 \\
\hline $\begin{array}{l}\text { Capacidad para absorber, } \\
\text { chupar o retener... }\end{array}$ & 27 & 30 & 22,2 & 37,8 & 14 & 25 & 5 & 56 \\
\hline Confusas & 8 & 8,9 & 13,3 & 4,4 & 2 & 5 & 0 & 0 \\
\hline $\mathrm{NS} / \mathrm{NC}$ & 2 & 2,2 & 4,4 & 0 & 0 & 0 & 0 & 0 \\
\hline Total respuestas & 90 & & 45 & 45 & 57 & 20 & 21 & 16 \\
\hline
\end{tabular}

Pregunta $F: ¿ C u a ́ l$ de los tres materiales es más permeable? ¿Cómo podrías definir la permeabilidad?

El objetivo conjunto de estas preguntas era valorar la coherencia o consistencia de las respuestas a la vez que se demandaba implícita (F1) o explícitamente (F2) una definición del concepto analizado. En las tablas II y III señalamos la respuesta que debería considerarse como «correcta», desde el punto de vista científico.

Pregunta $F(1):$ ¿Cuál de los tres materiales es más permeable?: El análisis conjunto de la muestra indica que dos de cada tres estudiantes consideran que el material más permeable es el A (grava gruesa). Sin embargo, el análisis por separado indica que, en el grupo M2, sólo la mitad de los estudiantes dan la respuesta correcta (Tabla II). Los grupos de control C1 y C3 (sin instrucción previa) muestran resultados similares a M2. En el grupo de control C2 (con instrucción previa) más del $80 \%$ de los estudiantes responde correctamente.

Pregunta $F(2)$ : ¿Cómo podrías definir la permeabilidad?: La mayoría de los estudiantes dan una respuesta correcta (Tabla III). Las respuestas son de dos tipos: 1) «Es la capacidad de un material para dejar pasar un líquido a través del mismo». 2) «Es la capacidad de un cuerpo o material para absorber (chupar o retener) un lí- quido...». Hay que destacar que en el grupo M1, un gran número de estudiantes «acertaba» al señalar el material A como el más permeable, pero luego no todos eran capaces de definir el concepto. Por el contrario, en el grupo M2 es mayor el número de definiciones correctas frente a los frecuentes errores al señalar el material $\mathrm{C}$ como el más permeable. El grupo de control $\mathrm{C} 1$ muestra un $70 \%$ de respuestas correctas frente al $55 \%$ en la pregunta anterior, situación más chocante todavía que la que presenta M2. El grupo C2 presenta un $95 \%$ de definiciones correctas (superando ligeramente el número de aciertos en la pregunta anterior). Las definiciones del grupo C3 sobre permeabilidad son totalmente consistentes con las respuestas sobre el material más permeable (coinciden en todos los casos). Si eligen el material A como el más permeable, entonces se refieren a la permeabilidad como la capacidad para dejar pasar un líquido. Los que han elegido $\mathrm{C}$ como el más permeable hacen referencia a la capacidad de absorción.

Pregunta G: Busca alguna analogía de la vida cotidiana para ejemplificar el fenómeno o el concepto.

A partir de los grupos M1 y M2 se han obtenido 97 respuestas diferentes (algunos estudiantes citaban varios ejemplos). Los ejemplos aportados por los grupos $\mathrm{C} 1$, C2 y C3 (65 respuestas) son muy similares a los anterio- 
res. Vamos a destacar las respuestas mayoritarias (Tabla IV) presentando algunos ejemplos que citamos textualmente (entre comillas).

La mayoría de los estudiantes (42\%) asocia el concepto de permeabilidad con la ropa: chaqueta o jersey de lana, camiseta de algodón, chubasquero o impermeable, etc. (1) «Cuando llueve llevamos ropa (impermeable) que no posee ningún espacio ni hueco en su tejido, por eso no deja pasar el agua. En cambio, las prendas de algodón (permeables) dejan pasar el agua porque dejan muchos espacios libres.»

Sin embargo, esta asociación de ideas no siempre responde al significado científico del concepto. Por ejemplo: (2) «...una camiseta de algodón es más permeable porque absorbe más agua, frente a un chubasquero que no absorbe nada, la rechaza». Buena parte de los alumnos que ejemplifican el fenómeno-concepto con la ropa no han contestado correctamente a las preguntas anteriores.

En general, los alumnos que proponían como ejemplo la formación de charcos en el suelo $(11 \%)$ habían dado respuestas válidas en la pregunta anterior y utilizaban el término correctamente desde el punto de vista «geológico». Por ejemplo: (3) «Los suelos que son de grava no suelen presentar charcos cuando llueve, en cambio, los suelos más arcillosos sí retienen el agua en superficie y forman charcos, porque éstos son más impermeables que la grava». (4) «Cuando llueve, en el parque que tiene el suelo de arena, siempre hay charcos y tardan en desaparecer, sin embargo, en donde aparco el coche, que tiene el suelo de grava fina, en muy pocas ocasiones hay charcos.»
Aunque no en todos casos se cumplía lo anterior: (5) «Cuando no se quiere que un suelo se encharque cuando llueve se utiliza arena fina para cubrirlo. La arena absorbe el agua y no permite que se hagan charcos en el suelo. Un suelo con grava gruesa se encharcará rápidamente cuando llueva, porque la grava no absorbe el agua.»

Los ejemplos que hacían referencia a la relación entre la permeabilidad y los cauces de los ríos o las aguas subterráneas $(11 \%)$ presentaban lecturas muy dispares: (6) «En la costa, donde la arena de playa es muy fina, al caer el agua sobre ella es retenida en dicha arena sin que adquiera una gran profundidad. Sin embargo, en la montaña donde la tierra está formada por granos de mayor tamaño como calizas, el agua que cae sobre ella se filtra y penetra a gran profundidad formando ríos subterráneos.» (7) «Las orillas de los ríos son permeables y a través de ellas el agua del río aporta nutrientes a éstas para que crezcan las plantas». (8) «En los cauces de los ríos se ponen grandes bloques de grava para encauzar el curso del río (ya que no "chupan" el agua, ésta sale repelida y sigue el cauce).»

Por último existen algunos ejemplos que calificamos como «curiosos»: (9) «En la vida diaria, cuando utilizamos un recipiente con todo el cuerpo lleno de grietas para lavar lechuga o cualquier otro alimento, depende de cuál sea dejará pasar mayor cantidad de agua. La lechuga deja pasar mayor cantidad de agua que la pasta, que deja pasar menor cantidad de agua.» (10) «Un ejemplo de permeabilidad es el bañador que deja pasar el agua y aunque te metas en la piscina, te mojas...»

Tabla IV

R espuestas a la pregunta G: Busca alguna analogía de la vida cotidiana para ejemplificar el fenómeno o el concepto.

\begin{tabular}{|l|c|c|c|c|c|c|c|}
\hline \multicolumn{1}{|c|}{ Ejemplos } & $\begin{array}{c}\text { Número } \\
\text { de respuestas } \\
\text { (M1 + M2) }\end{array}$ & \% M1 & \% M2 & $\begin{array}{c}\text { Número } \\
\text { de respuestas } \\
\text { (C1 + C2 + C3) }\end{array}$ & \% C1 & \% C2 & \% C3 \\
\hline $\begin{array}{l}\text { Ropa/paraguas/ } \\
\text { chubasquero }\end{array}$ & 41 & 52,1 & 32,6 & 34 & 66,6 & 50 & 40 \\
\hline $\begin{array}{l}\text { Formación de charcos } \\
\text { en el suelo }\end{array}$ & 11 & 10,4 & 12,2 & 6 & 4,8 & 8,3 & 15 \\
\hline $\begin{array}{l}\text { Cauces y aguas } \\
\text { subterráneas }\end{array}$ & 11 & 12,5 & 10,2 & 2 & 4,8 & 4,2 & 0 \\
\hline $\begin{array}{l}\text { Suelos de los cultivos } \\
\text { y macetas con plantas }\end{array}$ & 8 & 4,2 & 12,2 & 3 & 4,8 & 8,3 & 0 \\
\hline Esponja & 6 & 2,1 & 10,2 & 5 & 4,8 & 8,3 & 10 \\
\hline Filtros (s.1.) & 4 & 4,2 & 4,1 & 1 & 0 & 0 & 5 \\
\hline Cafetera y colador & 4 & 2,1 & 6,1 & 4 & 0 & 12,5 & 5 \\
\hline Células (ósmosis) & 2 & 0 & 4,1 & 0 & 0 & 0 & 0 \\
\hline Otros (1 ejemplo) & 4 & 8,3 & 4,1 & 6 & 14,3 & 0 & 15 \\
\hline Confusos & 2 & 0 & 4,1 & 4 & 0 & 8,3 & 10 \\
\hline NS/NC & 4 & 8,3 & 0 & 0 & 0 & 0 & 0 \\
\hline Total ejemplos & $\mathbf{9 7}$ & $\mathbf{4 8}$ & $\mathbf{4 9}$ & $\mathbf{6 5}$ & $\mathbf{2 1}$ & $\mathbf{2 4}$ & $\mathbf{2 0}$ \\
\hline
\end{tabular}




\section{INTERPRETACIÓN DE LOS RESULTADOS}

A la vista de los resultados obtenidos es obvio que el término permeabilidad presenta importantes dificultades conceptuales en estudiantes de nivel universitario. Un 40\% de los estudiantes del estudio no responde correctamente a la pregunta ¿qué material es más permeable? Un 30\% de los mismos no es capaz de formular una definición acertada del concepto, relacionando directamente, en la mayoría de estos casos, permeabilidad con absorción. Por otra parte, la mayoría de los estudiantes relacionan directamente la permeabilidad (impermeabilidad) con una propiedad de los tejidos de uso cotidiano, en menor medida con los materiales o procesos geológicos y, escasamente, con procesos biológicos.

Diversos autores han investigado en los últimos años sobre las dificultades conceptuales en el aprendizaje de la ciencia (Gil, 1983; Pozo, 1993; Prieto y Blanco, 1997; Pozo y Gómez Crespo, 1997). Estos últimos autores indican cuatro modelos respuesta ante las dificultades de los estudiantes para la comprensión de la ciencia: $a$ ) no aprenden porque no quieren aprender (motivación); $b$ ) no aprenden porque no tienen capacidad intelectual (desarrollo cognitivo); $c$ ) no aprenden porque no tienen conocimientos adecuados (ideas previas); d) no aprenden porque interpretan el mundo desde modelos distintos a los de la ciencia (cambio conceptual).

En el caso de los estudiantes de magisterio, podríamos «descartar» los dos primeros modelos como respuesta a nuestro problema, ya que, tanto la motivación como la capacidad intelectual se asumen como inherentes al estudiante universitario (aunque podría ser discutido). Por tanto, la explicación a las dificultades conceptuales debería buscarse en la existencia de conocimientos inadecuados, procedentes de niveles formativos previos, $\mathrm{o}$ en un modelo conceptual distinto del científico.

La coincidencia en las ideas alternativas que los alumnos poseen sobre este concepto nos lleva a suponer que el principal obstáculo aparece en los niveles formativos previos. Aunque muchos de estos estudiantes proceden del modelo educativo anterior a la LOGSE (EGB, BUP, COU), entendemos que los contenidos no han cambiado drásticamente y que las futuras generaciones de estudiantes universitarios arrastrarán los posibles «errores» generados dentro del actual marco formativo. Por ello, y paralelamente a este trabajo, estamos realizando un análisis de diferentes libros de texto de educación primaria, secundaria obligatoria y bachillerato (en preparación). Algunos de los resultados de este análisis, sobre libros de los últimos diez años, son adelantados en este trabajo y pueden ayudar a explicar algunas de las ideas alternativas así como las dificultades de aprendizaje detectadas.

\section{Consideraciones sobre la permeabilidad en los ni- veles formativos previos}

\section{Ejemplos de libros de texto de educación primaria}

Los libros de texto de primaria presentan definiciones y ejemplos muy diversos y, en ocasiones, bastante alejados del significado «científico» del concepto de permeabili$d a d$. De esta manera, encontramos autores que definen la permeabilidad como «capacidad de dejar pasar el agua» y otros como «capacidad de absorber el agua».

- Editorial P1. Curso: $3^{\circ}$ EP

«Los materiales que dejan pasar el agua se llaman permeables. Los que no la dejan pasar se llaman impermeables.»

\section{- Editorial P2. Curso: $4^{\circ}$ EP}

«Existen materiales que son impermeables, es decir, que no dejan pasar el agua, y otros que se llaman permeables, porque sí la dejan pasar.»

\section{- Editorial P3. Curso: $3^{\circ} \mathrm{EP}$}

«El papel secante, la esponja y la tiza también absorben fácilmente el agua. Son materiales permeables. Otros materiales, como el plástico, no absorben el agua, resbala sin penetrar en ellos porque son impermeables.»

\section{- Editorial P4. Curso: $3^{\circ} \mathrm{EP}$}

«Cuando los materiales se ponen en contacto con el agua se comportan de distintas formas. Algunos dejan pasar el agua a través de ellos, como la esponja: son materiales permeables, pues absorben el agua.»

«Pero existen otros materiales por los que no pasa el agua, como el plástico; son materiales impermeables, pues no absorben agua.»

\section{Ejemplos de libros de texto de educación secundaria obligatoria}

En los libros de texto de educación secundaria obligatoria las explicaciones son bastante más complejas y, en general, introducen el concepto de permeabilidad dentro de una serie de propiedades de las rocas y suelos, que explicarían diferentes comportamientos de los mismos ante los procesos naturales. No obstante, no aparece un significado común del concepto en los distintos textos. Para algunos autores, la permeabilidad está relacionada con la capacidad de retener agua. Para otros, es la capacidad de dejar circular el agua a través de ellas, aunque algunos también introducen el concepto retención de agua.

\section{- Editorial S1. Curso: $2^{\circ}$ ESO}

«Suelo arenoso: está formado por partículas grandes y abundantes (85\% de arena). Son suelos muy permeables, ya que las partículas están sueltas y, por tanto, no retienen el agua.»

«Suelo arcilloso: está compuesto por partículas muy pequeñas (70\% de arcilla). Tiene unos poros muy pequeños, retiene mucha agua, siendo muy poco permeable.»

- Editorial S2. Curso: $2^{\circ}$ ESO

«La permeabilidad de las rocas es la capacidad que tienen éstas de dejar circular el agua a través de ellas. Normalmente se debe a la existencia de poros. En las rocas con partículas tamaño arena o mayor, los poros están comunicados permitiendo la circulación del agua 
(areniscas, conglomerados), sin embargo, las rocas arcillosas aunque son capaces de retener gran cantidad de agua, no son permeables porque el tamaño de sus poros es muy pequeño.»

\section{Ejemplos de libros de texto de bachillerato}

En general, los libros de texto de bachillerato presentan definiciones bastante aproximadas al modelo científico de permeabilidad. Los ejemplos que encontramos hacen referencia a rocas y suelos y, en especial, a los procesos de percolación en superficie y circulación freática. En algunos casos, como en el ejemplo mostrado, intentan aclarar los conceptos que podrían presentar dificultades (acuífero, permeabilidad, entre otros). Si lo consiguen o no, depende del receptor de la información y de su modelo conceptual.

\section{- Editorial B1. Curso: $2^{\circ}$ Bachillerato}

«El agua que se infiltra, una vez que se llenan los poros del suelo, pasa hacia el sustrato rocoso y desciende por gravedad a través de los huecos de las rocas hasta una zona donde los poros están saturados de agua, formando así un acuífero; para que el agua pueda penetrar en las rocas se necesita que éstas sean permeables.»

Conceptos: «Los acuíferos son formaciones geológicas con agua que puede moverse o permanecer estanca. Los acuíferos pueden estar en rocas permeables (ejemplo areniscas) o en grietas $\mathrm{u}$ oquedades de rocas impermeables (ejemplo calizas).»

Permeabilidad: «Es la velocidad de infiltración del agua en el suelo saturado. La infiltración se produce por gravedad y, lógicamente, cuanto más huecos haya en el suelo y más grandes sean, su permeabilidad será mayor.»

\section{CONSIDERACIONES FINALES}

El modelo conceptual de permeabilidad que presenta un gran porcentaje de los estudiantes objeto de este trabajo se aleja bastante del modelo científico de permeabilidad. Para muchos estudiantes no queda claro qué es más o menos permeable y, menos todavía, por qué. El significado que se le atribuye finalmente se aleja de la acepción semántica y científica del término. Buena parte de los estudiantes considera que la permeabilidad de un material corresponde a la «capacidad de absorción» del mismo, frente a una definición científica del tipo «capacidad para dejar pasar un fluido». En muchos casos, los modelos conceptuales que los estudiantes presentan sobre el tema no son consistentes y muestran incoherencias al llegar a respuestas incompatibles ante la misma pregunta planteada de diferente forma o al demandarles una ejemplificación relacionada con el concepto.

Una explicación a este problema se puede encontrar, por un lado, en el modelo de permeabilidad (permeable, impermeable) que presentan los libros de texto citados anteriormente, especialmente en educación primaria y, por otro, en el uso que se hace del mismo en la vida cotidiana y, en ocasiones, incluso trivializado (por ejemplo, en la ropa o en los materiales de higiene íntima, aunque entraríamos en otra discusión). Probablemente por ello, ni siquiera se trata de una «transposición didáctica consciente» del concepto científico por parte de los autores de los textos de primaria.

Como indica Chevallard (1998), «la noción de distancia (entre dos puntos) se utiliza espontáneamente desde siempre», posteriormente se introdujo como concepto matemático y, más tarde, como objeto de enseñanza en las matemáticas. Del mismo modo, la permeabilidad o impermeabilidad es una cualidad de los materiales de uso cotidiano, es decir, estaba ahí y utilizamos el término sin pensar en su significado y en la causa de esta cualidad. Luego se convirtió en un concepto científico, un tanto difuminado, y más tarde en un complejo objeto de enseñanza en física, biología o geología, por poner algunos ejemplos. Mientras tanto, los estudiantes siguen preguntándose el para qué de las cosas (el impermeable sirve para no mojarse), en menor medida el porqué «visible» (porque no deja pasar el agua) y muy pocas veces el porqué «escondido» (porque el espacio entre las fibras que componen el tejido es muy pequeño).

A la vista de la dificultad de un concepto de uso cotidiano como permeabilidad y de las ideas alternativas que sobre el mismo presentan muchos estudiantes de nivel universitario (compartidas, resistentes e incoherentes desde el punto de vista científico), queda por hacer un análisis más profundo del problema. Por un lado, de las ideas y los modelos conceptuales de los alumnos de distintos niveles. Por otro, del uso del concepto en la vida cotidiana y del tratamiento del mismo en cada uno de los niveles educativos, es decir, de la transposición didáctica del concepto científico.

\section{AGRADECIMIENTOS}

Agradecemos a B. Martínez, M. de la Gándara, M.J. Gil y a dos revisores anónimos, los comentarios y sugerencias que han contribuido a mejorar la versión final de este trabajo. 


\section{REFERENCIAS BIBLIOGRÁFICAS}

CHEVALLARD, Y. (1998). La transposición didáctica. Del saber sabio al saber enseñado. Buenos Aires: Aique.

CUSTODIO, E. y LLAMAS, M.R. (1976). Hidrología subterránea. Barcelona: Omega.

DRIVER, R. (1988). Un enfoque constructivista para el desarrollo del currículo de ciencias. Enseñanza de las Ciencias, 6(2) pp. 109-120.

DRIVER, R., GUESNE, E. y TIBERGHIEN, A. (1989). Ideas científicas en la infancia y la adolescencia. Madrid: MECMorata.

FURIÓ MÁS, C.J. (1996). Las concepciones alternativas del alumnado en ciencias: dos décadas de investigación. Resultados y tendencias. Alambique: Didáctica de las Ciencias Experimentales, 7, pp. 7-17.

GIL, D. (1983). Tres paradigmas básicos en la enseñanza de las ciencias. Enseñanza de las Ciencias, 1(1), pp. 26-33.

KRNEL, D., WATSON, R. y GLAZAR, S. (1998). Survey of research related to the development of the concept of «matter». International Journal of Science Education, 20 (3), pp. 257-289.

LILLO, J. (1983). Como fomentar los principios de actividad y creatividad al aplicar el método científico en la escuela. Un ejemplo para la formación de maestros. Enseñanza de las Ciencias, 1(3), pp. 193-197.

POZO, J.I. (1993). Psicología y didáctica de las ciencias de la naturaleza, ¿concepciones alternativas? Infancia y aprendizaje, 62-63, pp. 187-204.

POZO, J.I. (1996). Las ideas del alumnado sobre la ciencia: de dónde vienen, a dónde van... Y mientras tanto qué hacemos con ellas. Alambique: Didáctica de las Ciencias Experimentales, 7, pp. 18-26.

POZO, J.I. y GÓMEZ CRESPO, M.A. (1997). ¿Qué es lo que hace difícil la comprensión de la ciencia? Algunas explicaciones y propuestas para la enseñanza, en Del Carmen, L. (coordinador). La enseñanza y aprendizaje de las ciencias de la naturaleza en educación secundaria, pp. 73-105. Barcelona: ICE, Universitat de Barcelona - Horsori.

POZO, J.I. y GÓMEZ CRESPO, M.A. (1998). Aprender y enseñar ciencia. Madrid: Morata.

PRICE, M. (1996). Introducing groundwater. Londres: Allen \& Unwin.

PRIETO, T. y BLANCO, A. (1997). Las concepciones de los alumnos y la investigación en didáctica de las ciencias. Colección Estudios y Ensayos. Málaga: Servicio de Publicaciones de la Universidad de Málaga - Centro de Profesores de Málaga.

PRIETO, T., BLANCO, A. y GONZÁLEZ, F. (2000). La materia y los materiales. Didáctica de las Ciencias Experimentales, 2. Madrid: Síntesis.

REAL ACADEMIA ESPAÑOLA (2001). Diccionario de la Lengua Española, 22a. ed. Madrid: Espasa.

YUS RAMOS, R. y REBOLLO BUENO, M. (1993). Aproximación a los problemas de aprendizaje de la estructura y formación del suelo en el alumnado de 12 a 17 años. Enseñanza de las Ciencias, 11 (3), pp. 265-280.

YUS RAMOS, R. (1994). «Balsas de agua y ríos subterráneos». Representaciones de los alumnos sobre la circulación freática. Su tratamiento en la educación secundaria.Enseñanza de las Ciencias de la Tierra, vol. extra, pp. 76-79.

\section{ANEXO}

\section{Libros de texto selecionados}

Antón, J.L., Barrio, J., Belart, C., Belart, A. y Pallarés, N. (1996). Ciencias de la naturaleza. $1^{o}$ ESO. Madrid: Editex.

Batllorí, R., Espinet, M., Franch, J., Gavaldà, A., Mollà, J., Pagès, J. y Santisteban, J. (1993). Enclave. Conocimiento del medio. $3^{\circ}$ Educación Primaria. Madrid: Bruño, 191 pp.

Cabrera, M.E., De Hoyos, C., Ledesma, J.L., Nieto, J.M., Revuelta, J.L., Romero, T., Salamanca, C., Torres, M.D. y Velasco, J.M. (1996). Ciencias de la Tierra y del medio ambiente, $2^{\circ}$ Bachillerato. Madrid: Editex, $497 \mathrm{pp}$.

Cintas, R. y Varo, R. (1993). Conocimiento del medio. $4^{\circ}$ Educación Primaria. Proyecto Albanta. Madrid: Alhambra Longman, $214 \mathrm{pp}$.

Jiménez, E. y Blázquez, T. (1993). Conocimiento del medio. $3^{\circ}$ Educación Primaria. Proyecto Trineo, Equipo Cauria. León: Everest, $237 \mathrm{pp}$.

Leal, A., García-Doncel, R., Aréjula, F., García, R., Montañés, A.M., Blanco, L. y Fernández, T. (1996). Ciencias de la naturaleza. $2^{\circ}$ ESO. Madrid: McGraw Hill, 263 pp.

Sarrión, J., Sánchez, R. y Pinto, D. (1993). Conocimiento del medio. $3^{\circ}$ Educación Primaria. Zaragoza: Edelvives, 167 pp. 